\title{
The Magnetic Memory Effect of Ferromagnetic Materials in the Process of Stress-Magnetism Coupling
}

\author{
Guoqing Wang, Ping Yan, Liwa Wei, and Zilong Deng \\ School of Mechanical Engineering, Liaoning Shihua University, Liaoning Province 113001, China \\ Correspondence should be addressed to Guoqing Wang; wangguoqing@lnpu.edu.cn
}

Received 24 April 2017; Accepted 16 July 2017; Published 14 August 2017

Academic Editor: Michele Zappalorto

Copyright ( 2017 Guoqing Wang et al. This is an open access article distributed under the Creative Commons Attribution License, which permits unrestricted use, distribution, and reproduction in any medium, provided the original work is properly cited.

\begin{abstract}
Ferromagnetic materials can produce the magnetic memory effect under stress. This provides a practical method to measure stress concentration. The relation between stress and magnetic characteristic is analyzed through energy balance theory. Forcemagnetism coupling process of Fe-C crystal system is simulated by CASTEP software which is based on first principle. Electron band structure, electron density of states, and atomic magnetic moment in the process of force-magnetism coupling process are calculated. Experimental investigation of the magnetic memory effect of ferromagnetic material under different stresses has been undertaken in X52 pipeline. The results show that the magnetic characteristic of ferromagnetic material weakens under stress, and the magnetic memory signals intensity linearly decreases with the increasing stress. When material yields, the variation character of magnetic memory signals suddenly changes and the inflection points of the stress- $B$ curves emerge. Experimental investigation is in agreement with the theoretical analysis.
\end{abstract}

\section{Introduction}

The ferromagnetic materials widely used in engineering structure have good mechanical properties and exhibit good ductility and toughness under external stress. It can effectively resist stress and deformation and ensure the safe operation of the equipment. However, the defect or discontinuity and other factors often existent in the metal will produce the stress concentration which induces the local large deformation or plastic deformation [1]. The plastic deformation is the early stage of materials damage under stress and always becomes the source of materials failure. So, it can prevent and reduce the damage accidents through detecting the stress concentration and plastic deformation which is induced by stress concentration in engineering field.

The magnetic memory effect is the change of ferromagnetic materials' magnetization characteristics under the stress in the weak magnetic field of the earth, and when the stress is removed, the change is irreversible [2]. It is a noncontact nondestructive testing method for stress concentration and plastic deformation [3-5]. However, the current research on the mechanism of magnetic memory effect of stress is far from conclusive [6-9]. The magnetic memory mechanism and signal characteristics are explained from different perspectives by a lot of researches, but there is no definite conclusion on them [10-14]. So the study on the mechanism of stress-magnetism coupling of ferromagnetic material and the characteristics of magnetic memory effect has aroused more and more concerns in many countries [15-17].

The present paper explores the magnetic memory effect of ferromagnetic materials under stress. Firstly, the characteristics of the magnetic memory signals on the surface of the ferromagnetic materials are analyzed from the point of view of the energy balance of the ferromagnetic body. Then the process of stress-magnetism coupling is simulated by CASTEP software based on the first principle. The influences of stress on the electron band structure, the electron density of states, and the atomic magnetic moment are analyzed. The quantitative relationship between the stress and the magnetic memory signals is obtained. In the last part, the ferromagnetic pipeline pressure experiments are carried out to verify the theoretical analysis of the magnetic memory effect of ferromagnetic materials under stress. 


\section{Mechanism Analysis}

The basic characteristics of ferromagnetic materials is the magnetic domain structure, and magnetic domain is the basis of all magnetic properties [18]. The essence of magnetic domain is an ordered arrangement of atomic magnetic moment in a small region, and its formation and existence are determined by interaction of various energies. The various energies are subject to the law of thermodynamics that the total free energy reaches minimum upon system equilibrium. Here, the magnetic memory signal characteristics which occur in the process of force and magnetic coupling are analyzed through the energy balance theory.

The magnetic domain will rotate when the external magnetic field $H$ is applied. Assume that the angle between the easy magnetization direction of magnetic domain and the direction of the magnetic field is $\theta_{0}$. When the magnetic moment is in the direction of easy magnetization, the energy of the external magnetic field $E_{H}$ of ferromagnetic magnet is

$$
E_{H}=-\mu_{0} M_{s} H \cos \theta_{0} .
$$

In the formula, $\mu_{0}$ is the vacuum permeability, $M_{s}$ is the saturation magnetization, and $H$ is the external magnetic field strength.

When subjected to external magnetic field, the magnetic moment will rotate a small angle, the energy of the external magnetic field $E_{H}^{\prime}$ of iron magnet is

$$
E_{H}^{\prime}=-\mu_{0} M_{s} H \cos \left(\theta_{0}-\theta\right)
$$

and $E_{H}^{\prime}$ is less than $E_{H}$. The greater $\theta$ is, the smaller the external magnetic energy is. If there are no other obstacles, the magnetic moment will continue to rotate until $\theta=\theta_{0}$. But the stress $\sigma$ which exists in a ferromagnetic material will produce stress energy $E_{\sigma}$ which can be expressed in the following formula:

$$
E_{\sigma}=\frac{3}{2} \lambda_{s} \sigma \sin ^{2} \theta
$$

In the formula, $\lambda_{s}$ is the magnetostrictive coefficient.

The existence of stress can produce the stress anisotropy, which is the same as the magnetic anisotropy, which hinders the rotation of the magnetic moment. At this time, the influence of the magnetization of the material should be considered, so the total free energy in the unit volume is the sum of the stress energy $E_{\sigma}$ and the external magnetic field energy $E_{H}^{\prime}$; that is,

$$
E=E_{\sigma}+E_{H}^{\prime}=\frac{3}{2} \lambda_{s} \sigma \sin ^{2} \theta-\mu_{0} M_{s} H \cos \left(\theta_{0}-\theta\right) .
$$

Formula (4) derivation can get relation between $\theta$ and $H$ :

$$
\frac{d E}{d \theta}=3 \lambda_{s} \sigma \sin \theta \cos \theta-\mu_{0} M_{s} H \sin \left(\theta_{0}-\theta\right)=0 .
$$

Under weak magnetic field, the magnetic moment rotation angle is very small, so in formula (5) $\sin \theta=\theta, \cos \theta \approx 1$, and $\sin \left(\theta_{0}-\theta\right) \approx \sin \theta_{0}$, so formula (5) can be simplified as

$$
\theta=\frac{\mu_{0} M_{s} H}{3 \lambda_{s} \sigma} \sin \theta_{0} \text {. }
$$

In addition, the magnetization in the direction of the magnetic field can be expressed as

$$
M=M_{s} \cos \left(\theta_{0}-\theta\right) .
$$

The initial magnetic susceptibility $\chi$ of the material is calculated by (6) and (7):

$$
\begin{aligned}
\chi & =\left(\frac{d M}{d H}\right)_{H \rightarrow 0}=M_{s} \sin \left(\theta_{0}-\theta\right) \frac{d \theta}{d H} \\
& \approx M_{s} \sin \theta_{0} \frac{d \theta}{d H}=\frac{\mu_{0} M_{s}^{2}}{3 \lambda_{s} \sigma} \sin ^{2} \theta_{0} .
\end{aligned}
$$

Formula (8) is the same direction, uniform stress region of the initial magnetic susceptibility. In fact, the ferromagnetic material is a multicrystal, and the stress direction is different in different grains. $\theta_{0}$ has a variety of values; therefore, it is needed to find out the average value of $\chi$ in different directions. Assume that the direction of stress is uniform in the domain, and the $\theta_{0}$ value in formula (8) is averaged, and the actual crystal magnetic susceptibility $\chi_{\text {(multi) }}$ is expressed as

$$
\begin{aligned}
\chi_{(\text {multi })} & =\bar{\chi}=\frac{\mu_{0} M_{s}^{2}}{3 \lambda_{s} \sigma} \cdot \frac{1}{4 \pi} \int_{0}^{2 \pi} \int_{0}^{\pi} \sin ^{2} \theta_{0} \sin \theta_{0} d \theta_{0} d \phi \\
& =\frac{\mu_{0} M_{s}^{2}}{3 \lambda_{s} \sigma} \cdot \frac{2}{3}=\frac{2 \mu_{0} M_{s}^{2}}{9 \lambda_{s} \sigma} .
\end{aligned}
$$

Formula (9) shows that the stress leads to the change of magnetic susceptibility and further leads to the change of the magnetization intensity in the local region. Under the action of the external magnetic field $H$, the relationship between the magnetic susceptibility and the magnetic flux density $B$ is as follows:

$$
B=\mu_{0}\left(1+\chi_{\text {(multi) }}\right) H=\left(\mu_{0}+\frac{2 \mu_{0}^{2} M_{s}^{2}}{9 \lambda_{s} \sigma}\right) H .
$$

Formula (10) shows that, in the areas of stress concentration, the significantly greater force will reduce the local magnetic flux density, and this leads to magnetic distortion in the local region, that is, the formation of magnetic memory signal.

Here, it should be noted that the stress $\sigma$ in formula (10) is equal to external stress $\sigma_{\text {ext }}$ when ferromagnetic materials are in the elastic deformation stage. While ferromagnetic materials start to yield, a lot of dislocations and plastic deformation that can reduce the actual stress within materials appear. At present, the stress $\sigma$ in formula (10) should be replaced by equivalent stress $\sigma_{\text {eff }}$. The relation between $\sigma_{\text {ext }}$ and $\sigma_{\text {eff }}$ is

$$
\sigma_{\text {eff }}=C \sigma_{\text {ext }} .
$$

In the formula, $C$ is a coefficient which is less than 1 and relates to the number of dislocations and the degree of plastic deformation.

According to the analysis above, the magnetic memory signals characteristic will change when ferromagnetic materials are in the state of plastic deformation. 


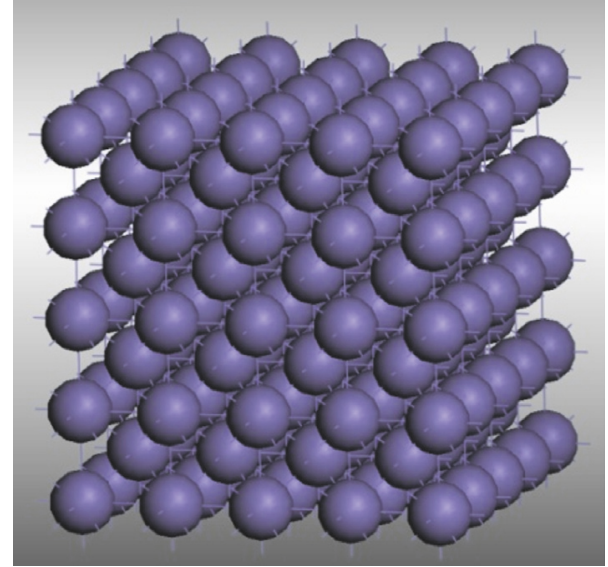

FIGURE 1: The force-magnetism coupling calculation model.

\section{Simulation of the Process of Stress-Magnetism Coupling}

Based on the theoretical analysis of the magnetic memory signals characteristics of ferromagnetic material under stress, the first principle method is used to simulate the magnetic memory effect in the process of force magnetic coupling. A model of the $\alpha$-Fe supercellular crystal with body centered cubic lattice structure was built by CASTEP module based on the first principle. The lattice constant in the ground state is set to $2.8664 \AA$ and, respectively, extended four units in the $a, b$, and $c$ three crystallographic directions. The $4 \times 4$ $\times 4$ supercell is established. In the actual steel, the carbon element is contained in the model, and the structure of the crystal model is formed by the replacement of the original cell structure. The crystal calculation model is shown as Figure 1.

In the calculation, the magnetic memory effect is related to the state of the electron spin motion, so the electron spin polarization is added. $K$ point sampling of the Brillouin zone is $15 \times 15 \times 15$, and the cut-off energy is calculated as $500 \mathrm{eV}$. The GGA (generalized gradient approximation) function is used to approximate the calculation process of the forcemagnetism coupling. The influences of external stress on the electron density and the gradient distribution of the system are considered in the GGA function. So the more accurate calculation can be obtained by the selection of GGA function [19].

3.1. System Energy. To simulate the actual water pressure state, the $X, Y$, and $Z$ three directions' tensile stresses are simultaneously applied on the established model. The system energy change in the calculation process is shown in Figure 2.

The external force will lead to the increase of system energy, and then the system state becomes unstable. The energy balance point is achieved through the multiple circulation calculation. It can be seen from Figure 2 that the system energy undergoes several fluctuations in the early stage and finally reaches a minimum value, and the system reached the stable state of energy balance. The results show that the calculation process is convergent.

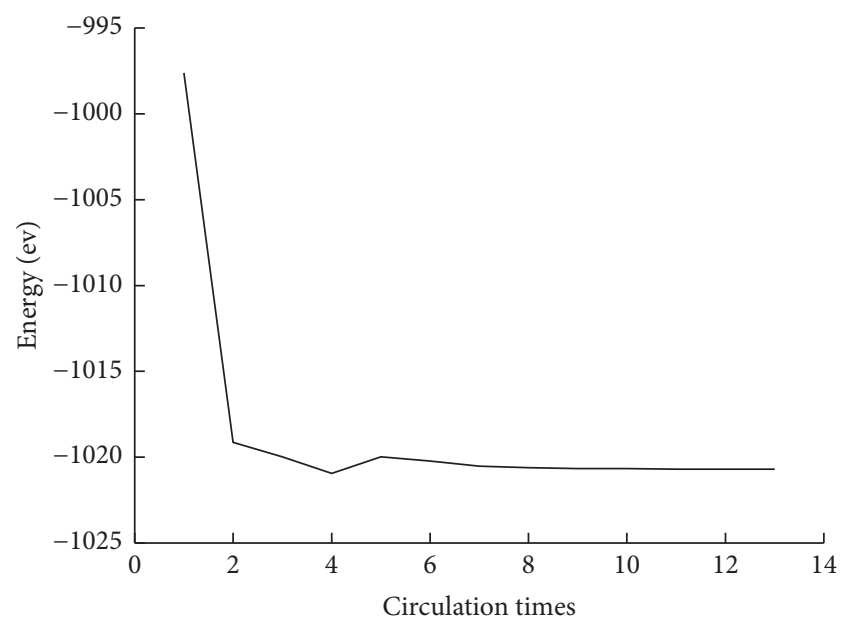

FIGURE 2: System energy.

3.2. Calculation of Electron Band Structure and Density of States. The magnetic properties of ferromagnetic material are determined by the atomic magnetic moment which is determined by the spin movement and distribution of electron. The crystal structure of ferromagnetic materials will vary under the action of external stress, and then the spin movement and distribution of orbital electrons outside atomic nucleus are influenced. That causes the variation of material magnetic characteristic. The variation can be analyzed through the electron band calculation and density of states distribution under different stress. The calculation results of electron band structure are shown in Figure 3.

It can be seen from Figure 3 that the electron band structure of ferromagnetic system in the Brillouin zone under different stresses are overlapped, and the localized and itinerant orbital electrons band are distributed near the Fermi level (the energy is zero). The electron band is bent and intersects with Fermi level. These features indicate that the material is ferromagnetic. The shapes of the energy band have no change under external stress within 0-20 MPa; that is, the material retains ferromagnetic characteristic. But the energy band distributions move far away from the Fermi level under external stress, and then the electron energy near Fermi level gradually decreases with the increasing stress, which will cause the variation of ferromagnetic characteristic of material.

The electron energy band distribution is related to the total electrons density of states which are the difference of density of states between spin up electrons and spin down electrons. In order to further analyze the influence of the stress on ferromagnetic characteristics, the total electron density states are calculated under different stresses. The calculation results are shown in Figure 4.

It can be seen from Figure 4 that, near the Fermi level, the peak values and valley values of the total electron density of states distribution curve significantly decrease under the external stress, which indicate that the numbers of the spin electron near the Fermi level gradually decrease and the ferromagnetic characteristic of material gradually weakens with the increasing external stress. 


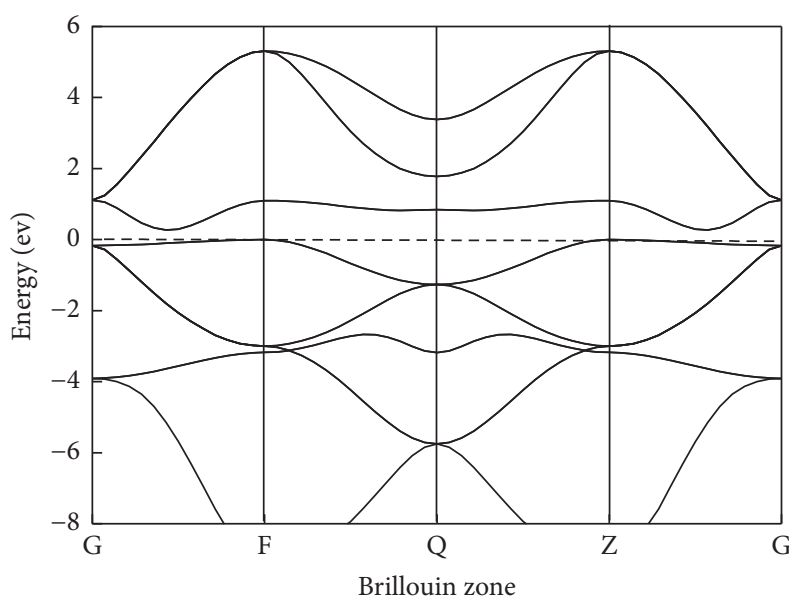

(a)

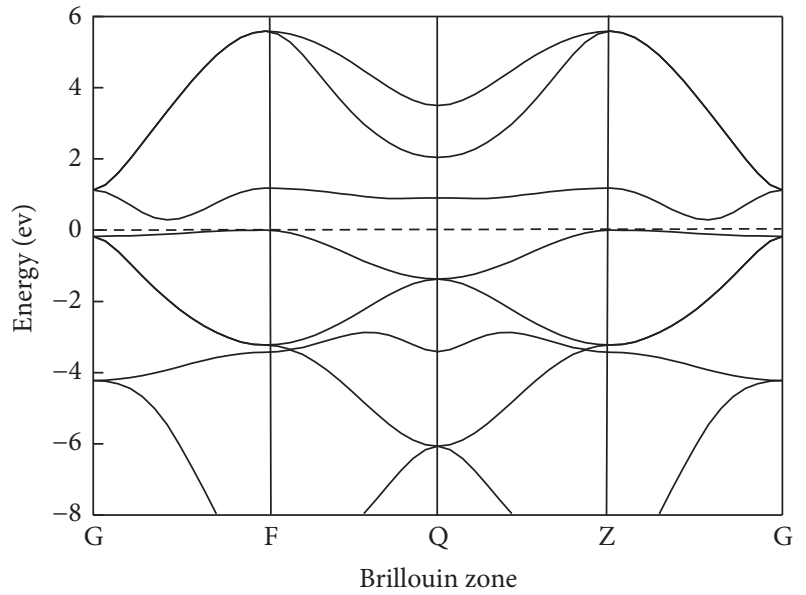

(c)

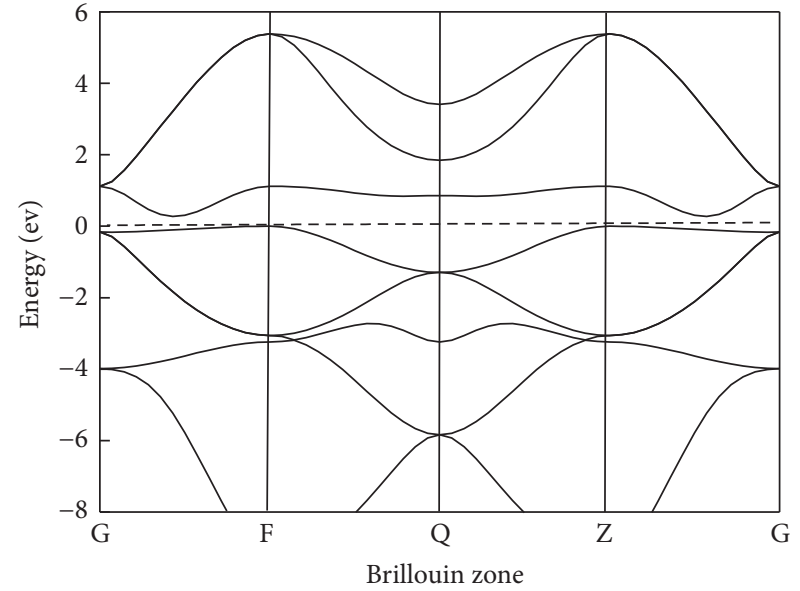

(b)

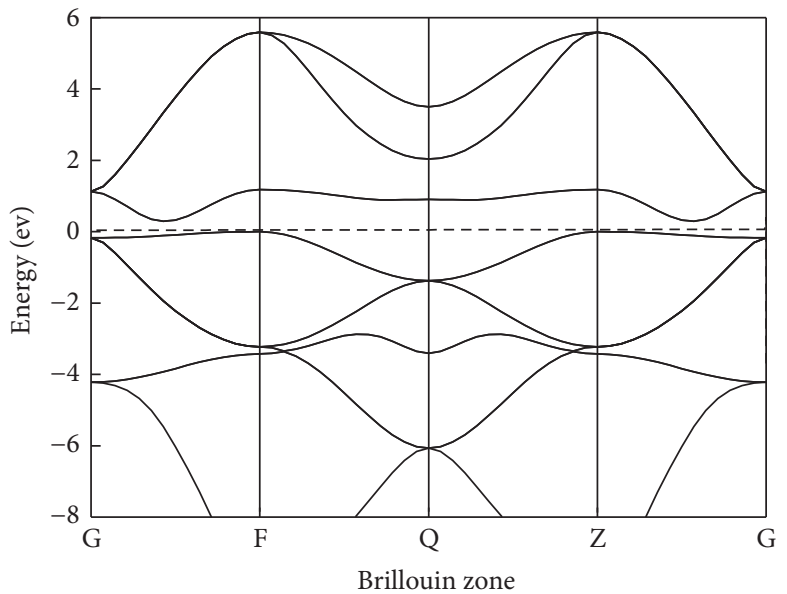

(d)

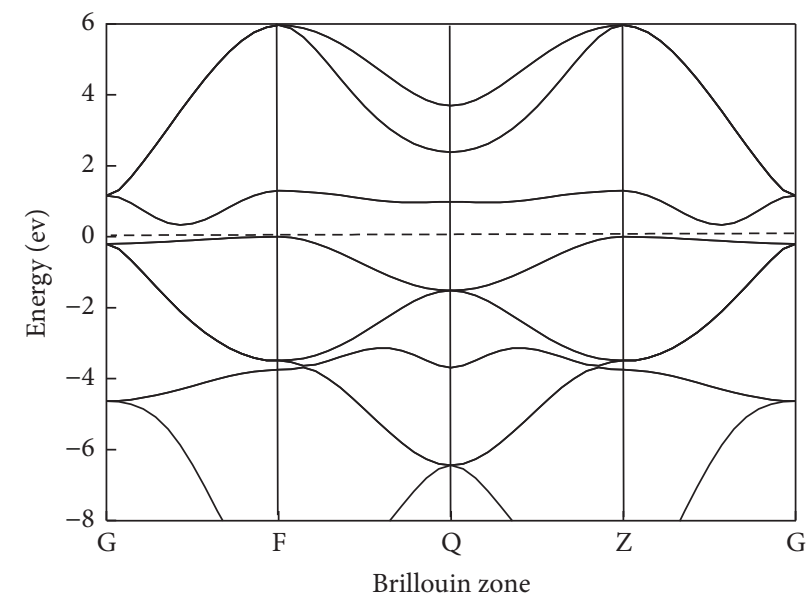

(e)

Figure 3: Electron band structure: (a) under 0 MPa stress; (b) under 5 MPa stress; (c) under $10 \mathrm{MPa}$ stress; (d) under $15 \mathrm{MPa}$ stress; (e) under $20 \mathrm{MPa}$ stress.

3.3. Calculation of Atomic Magnetic Moment. The variation features of the magnetic characteristics of ferromagnetic materials under different external stresses have been qualitatively analyzed by calculating the electron band structure and density of states. In order to qualitatively analyze the magnetic memory effect of ferromagnetic materials in the process of stress-magnetism coupling, the atomic magnetic moments of ferromagnetic materials under the external stresses within $0-20 \mathrm{MPa}$ are calculated, and the results are shown in Figure 5. 


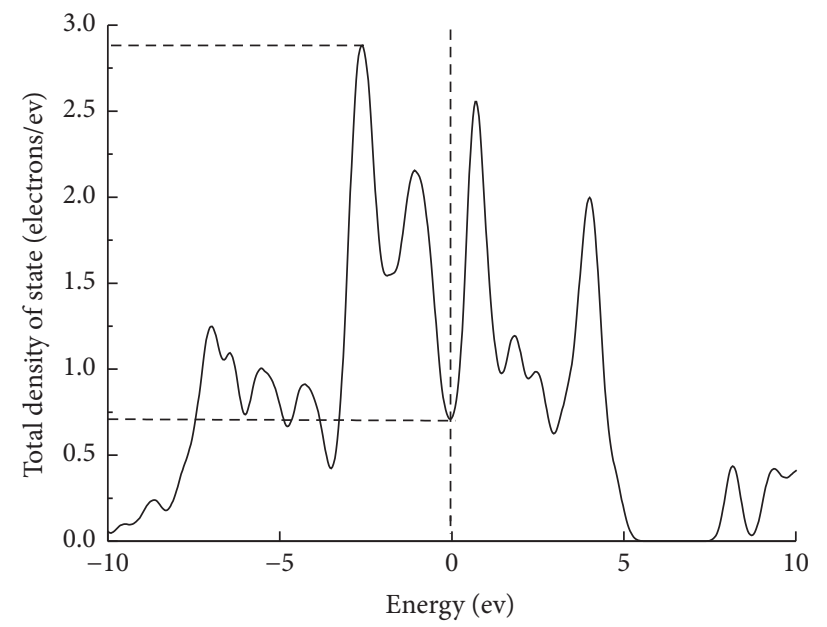

(a)

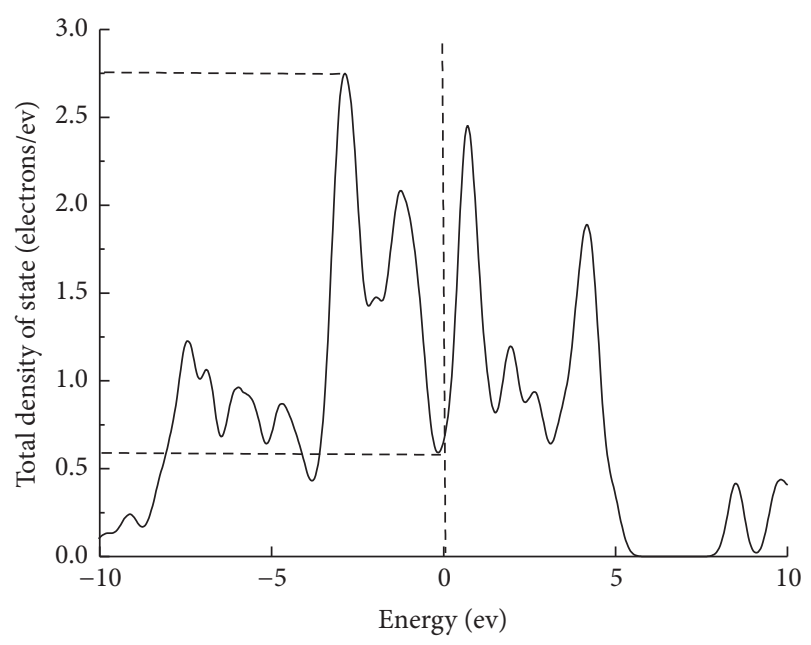

(c)

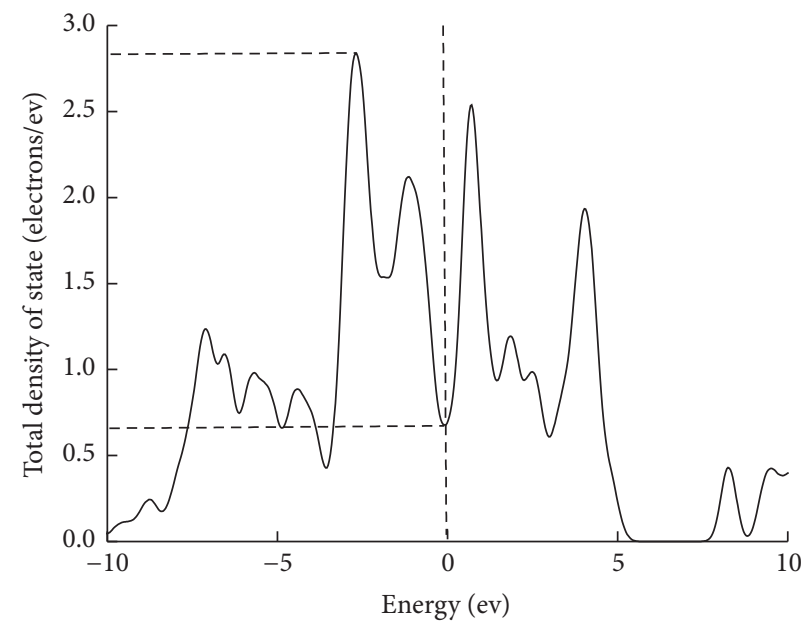

(b)

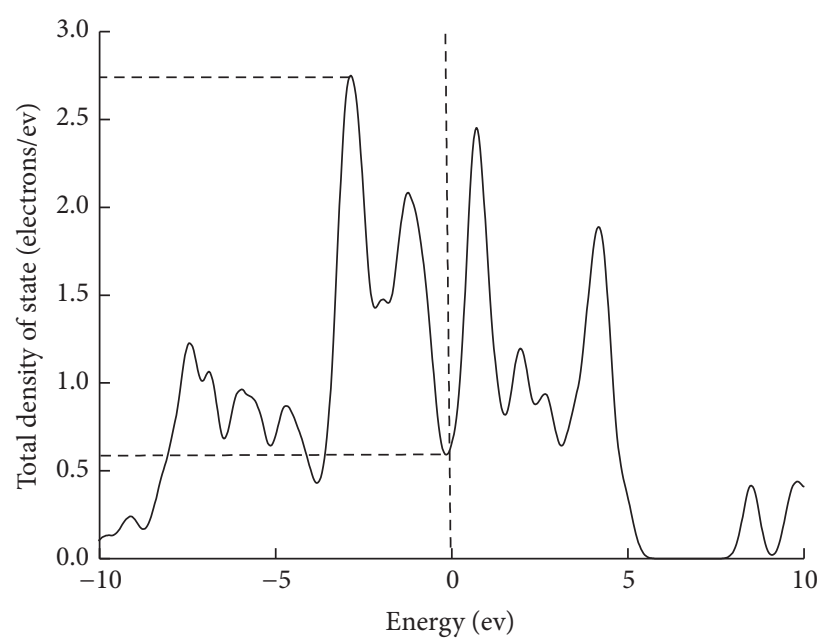

(d)

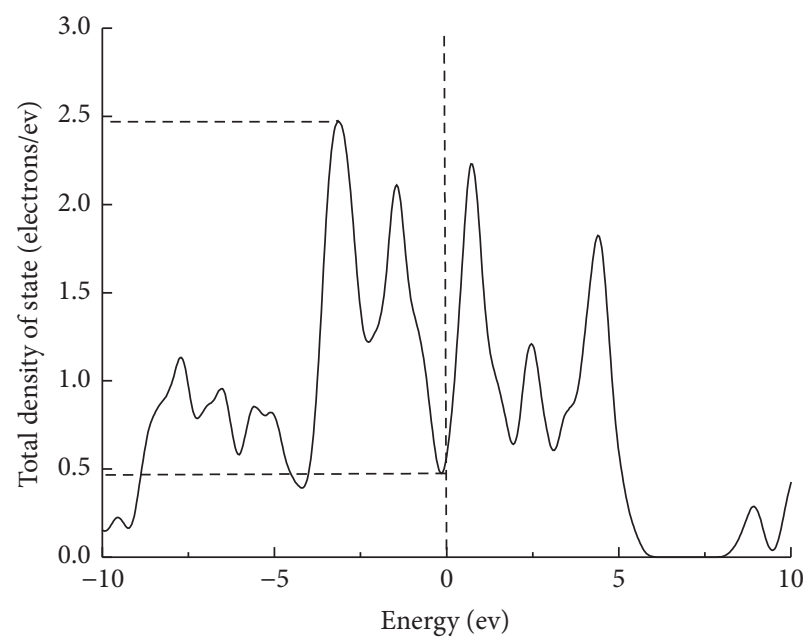

(e)

FIGURE 4: Electron density of states: (a) under 0 MPa stress; (b) under 5 MPa stress; (c) under $10 \mathrm{MPa}$ stress; (d) under $15 \mathrm{MPa}$ stress; (e) under $20 \mathrm{MPa}$ stress. 


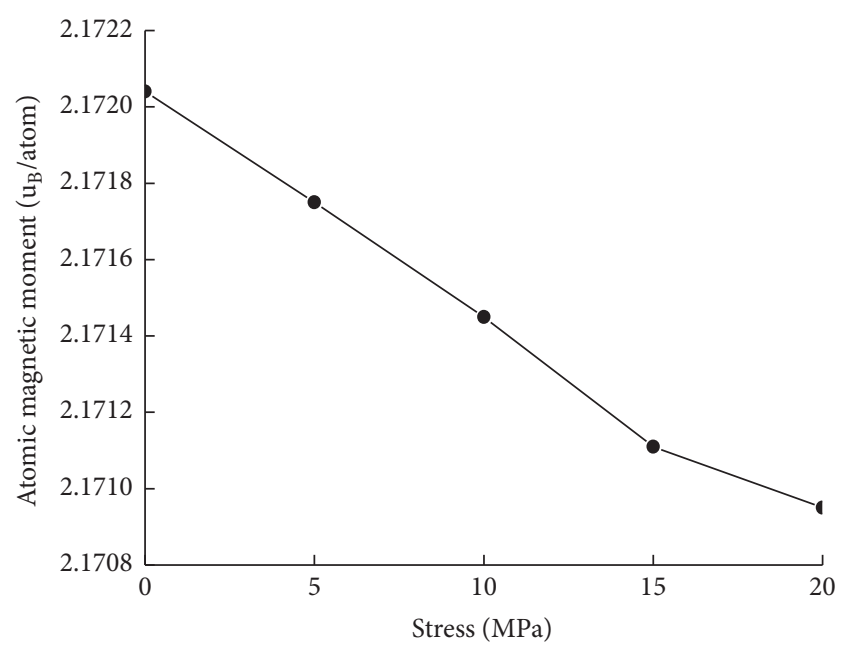

FIGURE 5: The relation between atomic magnetic moment and stress.

It can be seen from Figure 5 that the atomic magnetic moment linearly decreases with the increasing external stress at early stage and there is an inflection point in the curve when the stress increases to $15 \mathrm{MPa}$. The curve slope decreases under greater stress than $15 \mathrm{MPa}$.

The magnetization of materials is related to the atomic magnetic moment. The relationship between the atomic magnetic moment and magnetization intensity $M$ is as follows:

$$
M=\frac{\sum \mu_{j}}{V} .
$$

In the formula, $\mu_{j}$ is the atomic magnetic moment, $V$ is the solid volume, and $\sum \mu_{j}$ is the sum of all the atomic magnetic moments in solid.

Thus, the relationship between $\mu_{j}$ and the magnetic flux density $B$ is

$$
B=\mu_{0}(H+M)=\mu_{0}\left(H+\frac{\sum \mu_{j}}{V}\right) .
$$

According to the calculations and analyses above, the conclusion can be drawn that the magnetic flux density of ferromagnetic materials varies and there exists sudden change in the process of stress-magnetism coupling.

\section{Experimental Section}

4.1. Specimen Preparation. In order to study the magnetic memory signal characters of ferromagnetic materials in the process of stress-magnetism coupling, the pipeline pressure experiment is designed. The experiment material is ferromagnetic X80 steel which is applied widely in engineering. The external diameter of pipeline is $273 \mathrm{~mm}$ and the wall thickness is $6 \mathrm{~mm}$. To eliminate the influence of the edge effect produced by specimens with finite dimensions on weak magnetic signal measurement, the length of experimental pipeline is $120 \mathrm{~m}$. Signal measurement is carried out on the middle pipeline with the length range of $15 \mathrm{~mm}$. The strain gages monitored the stress-strain state of pipeline under different stress which are pasted on positions 1 and 2 which are shown in Figure 6. Weak magnetic signals sensors which are based on fluxgate and used to measure the magnetic field intensity in $X$ direction (normal direction) and $Y$ direction (axial direction) are fixed beside the strain gages. The measurement range of sensors is $0-100000 \mathrm{nT}$ and the measurement sensitivity is $1 \mathrm{nT}$.

4.2. Experimental Procedure. The two ends of the pipeline were sealed by high pressure blind plate. There were the pressure sensor which was used to monitor the pressure within the pipeline and the water injection gate which was connected to the pressure device. The strain changes were monitored and recorded during the process of pressure increases from zero. The pressure device stopped when inflection point of stressstrain curve emerged (i.e., the plastic deformation of pipeline emerged). The dates of stress-strain and magnetic memory signals were recorded in the whole process. Then the pressure within the pipeline drops to zero. To verify the reliability of the data, the experimental procedure was repeated two times.

4.3. Results and Discussion. In the three experimental procedures, the variation characteristics of stress-strain and magnetic memory signals in position 1 were the same as those in position 2. So, here, the dates only in position 1 are used to analyze the experimental results. The stress-strain diagram in three experimental procedures was shown in Figure 7.

It can be seen from Figure 7 that the strain of the pipeline linearly increases with increasing stress in the elastic deformation stage, and the relation between stress and strain is proportional. When the stress increases to $15 \mathrm{MPa}$, the inflection point of curve emerges in the first experimental procedure, which indicates that the materials start to yield and pipeline start to produce plastic deformation. The stresses corresponding to the inflection points of stress-strain curve which show materials yield are, respectively, $15.5 \mathrm{MPa}$ and $16 \mathrm{MPa}$ in the second and the third experimental procedure. The major reason for the increase of yield stress is that the plastic deformation causes the materials to harden. The magnetic memory signals testing results in three experimental procedures were shown in Figure 8.

It can be seen from Figure 8 that, in three experimental procedures, the magnetic flux density $B$ both in $X$ direction and in $Y$ direction linearly decreases with increasing stress, and the inflection points of the stress- $B$ curves emerge and the variation character of magnetic memory signals suddenly changes when the pipeline yields. In the plastic deformation stage, the slope of all the curves decreases and the variation rate of magnetic induction intensity decreases.

\section{Conclusion}

In the here presented paper, systematic investigation of magnetic memory effect of ferromagnetic material under stress has been performed via theory and experimental approaches. The primary cause of magnetic memory effect formation is that the stress causes the variation of the magnetic susceptibility and atomic magnetic moment of ferromagnetic material. The variety features of magnetic 


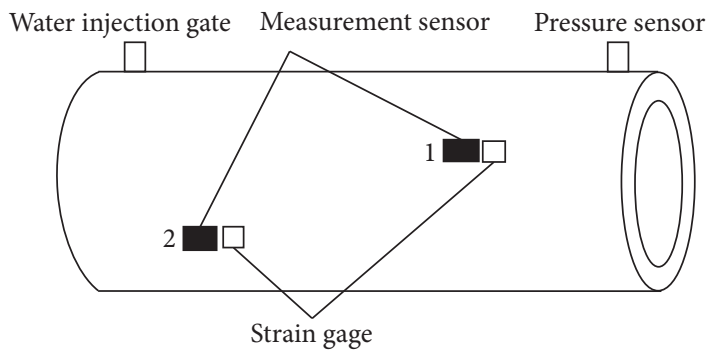

(a)

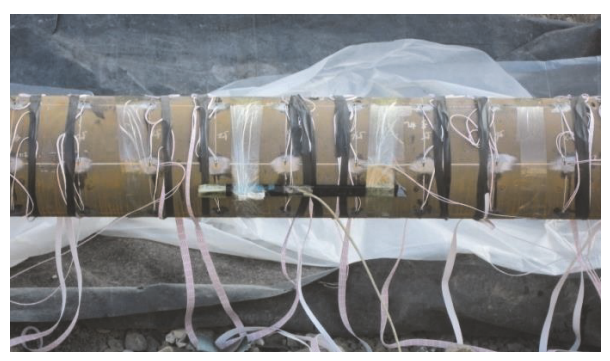

(b)

FIGURE 6: Experimental device: (a) the schematic diagram of experimental device. (b) The photograph in the scene.

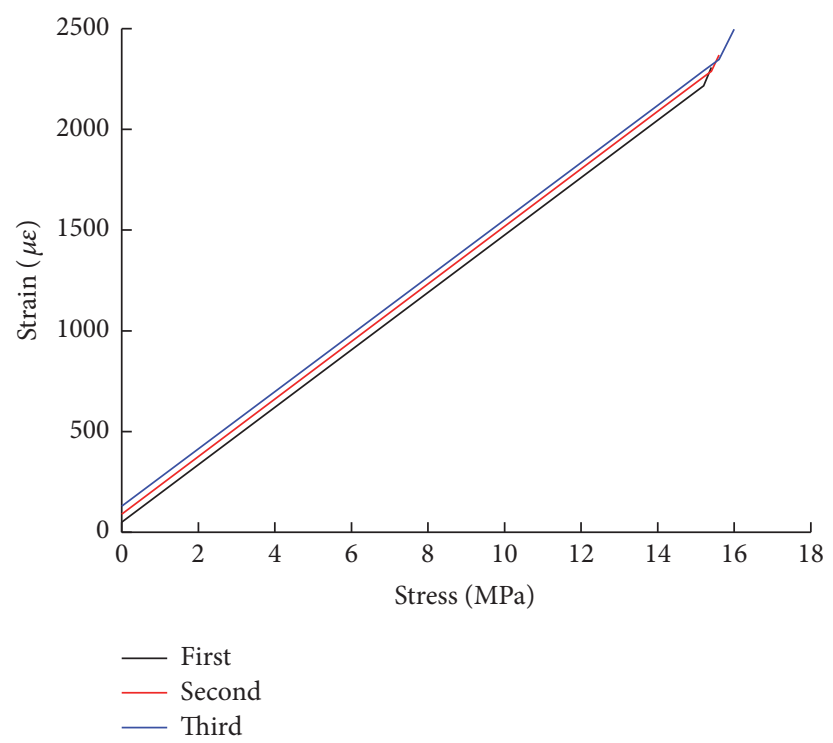

FIGURE 7: The stress-strain curve in three experimental procedures.

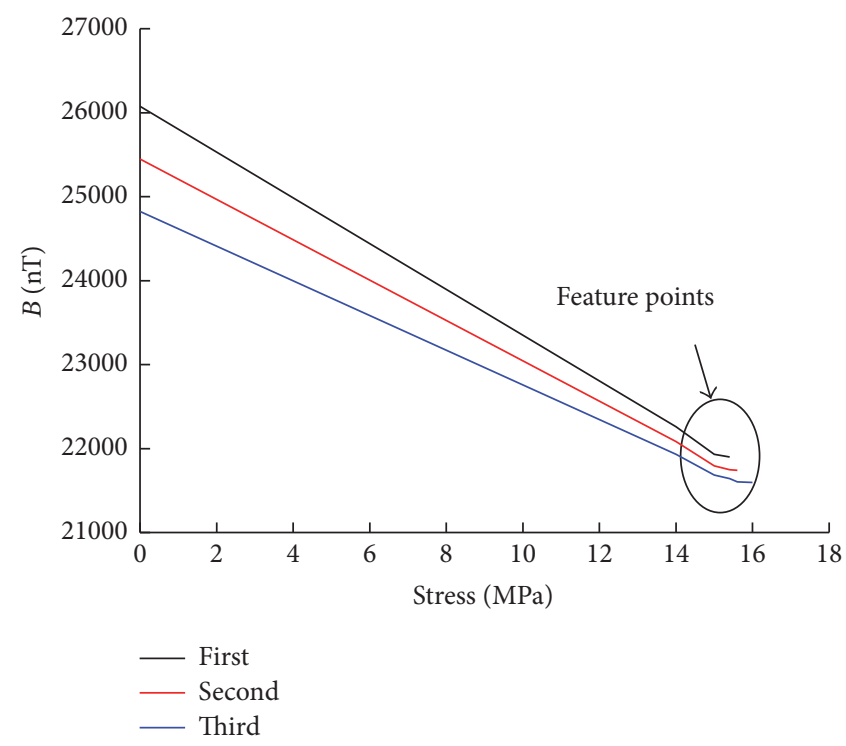

(a)

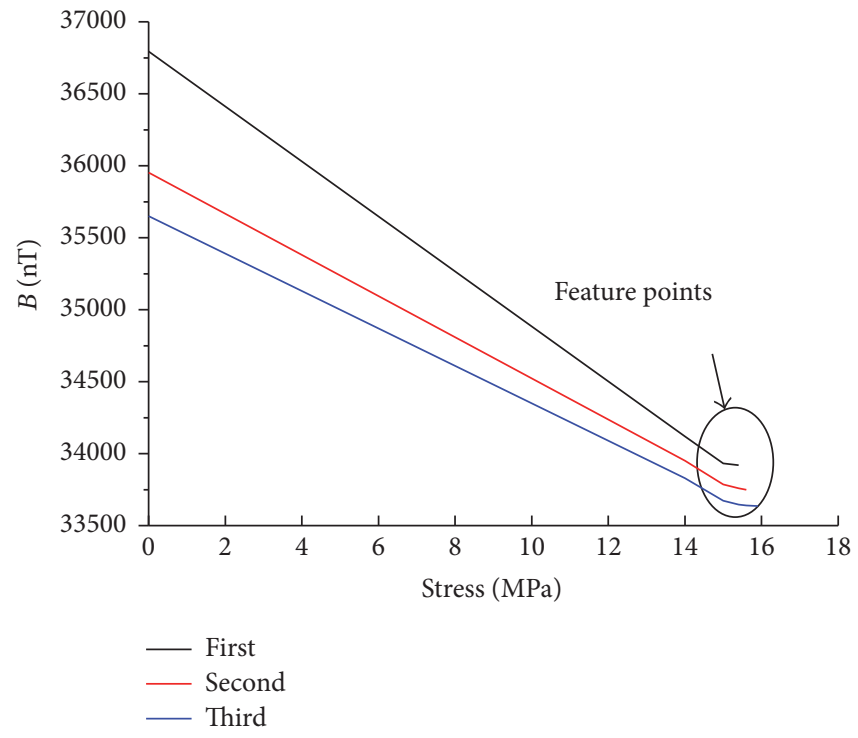

(b)

FIgURE 8: The signals character of magnetic memory in three experimental procedures: (a) The $X$ direction. (b) The $Y$ direction. 
characteristic are obtained by calculating the electron band structure, electron density of states, and atomic magnetic moment. It is significant that there is a linear relationship between stress and magnetic memory signals, and the stress$B$ curves have the inflection points which indicate that the materials yield and start to produce plastic deformation. The theory investigation is validated by the pressure experiment. This paper provides a convenient and practical approach to detecting the stress concentration and plastic deformation and predicting the damage of ferromagnetic material.

\section{Disclosure}

The founding sponsors had no role in the design of the study; in the collection, analyses, or interpretation of data; in the writing of the manuscript; and in the decision to publish the results.

\section{Conflicts of Interest}

The authors declare no conflicts of interest.

\section{Acknowledgments}

This work was funded by project supported by the National Natural Science Foundation of China (Grant no. 61571308) and project supported by Liaoning Education Department of China (Grant no. L2016009).

\section{References}

[1] S. Yoichi, "Stress Concentration Problems," Mathematical and Computational Analyses of Cracking Formation, vol. 2, pp. 1730, 2014.

[2] A. Dubov, A. Dubov, and S. Kolokolnikov, "Application of the metal magnetic memory method for detection of defects at the initial stage of their development for prevention of failures of power engineering welded steel structures and steam turbine parts," Welding in the World, vol. 58, no. 2, pp. 225-236, 2014.

[3] S. M. Kolokolnikov, A. A. Dubov, and A. Y. Marchenkov, "Determination of mechanical properties of metal of welded joints by strength parameters in the stress concentration zones detected by the metal magnetic memory method," Welding in the World, vol. 58, no. 5, pp. 699-706, 2014.

[4] R. Maciej, "Metal magnetic memory testing of welded joints of ferritic and austenitic steels," NDT \& E International, vol. 44, pp. 305-310, 2011.

[5] A. Dubov and S. Kolokolnikov, "The metal magnetic memory method application for online monitoring of damage development in steel pipes and welded joints specimens," Welding in the World, vol. 57, no. 1, pp. 123-136, 2013.

[6] C. Sara, M. Augusto, and U. Mariangela, "Modeling Ultrasounds for Nondestructive Testing Applications," in Ultrasonic Nondestructive Evaluation Systems, vol. 12, pp. 47-82, 2014.

[7] Sorabh, A. Gupta, and K. Chandrasekaran, "Finite Element Modeling of Magnetic Flux Leakage from Metal Loss Defects in Steel Pipeline," Journal of Failure Analysis and Prevention, vol. 16, no. 2, pp. 316-323, 2016.

[8] J. Jongwoo and L. Jinyi, "Non-destructive evaluation of cracks in a paramagnetic specimen with low conductivity by penetration of magnetic fluid," NDT \& E International, vol. 42, pp. 297-303, 2009.

[9] K. Yao, Z. D. Wang, B. Deng, and K. Shen, "Experimental Research on Metal Magnetic Memory Method," Experimental Mechanics, vol. 52, no. 3, pp. 305-314, 2012.

[10] B. Liu, W. Sun, and Y. Lin, "The Study of Electromagnetic Stress Testing Method on Oil-Gas Pipelines Based on WT," Geomaterials, vol. 04, pp. 55-63, 2014.

[11] H. Hauser, Y. Melikhov, and D. C. Jiles, "Examination of the equivalence of ferromagnetic hysteresis models describing the dependence of magnetization on magnetic field and stress," IEEE Transactions on Magnetics, vol. 45, no. 4, pp. 1940-1949, 2009.

[12] B. Liu, Y. Y. He, and H. Zhang, "Study on characteristics of magnetic memory testing signal based on the stress concentration field," IET Science Measurement \& Technology, vol. 11, pp. 2-8, 2017.

[13] M. Roskosz and P. Gawrilenko, "Analysis of changes in residual magnetic field in loaded notched samples," NDT \& E International, vol. 41, pp. 570-576, 2008.

[14] J. Leng, Y. Liu, G. Zhou, and Y. Gao, "Metal magnetic memory signal response to plastic deformation of low carbon steel," NDT \& E International, vol. 55, pp. 42-46, 2013.

[15] L. J. Yang, B. Liu, and L. J. Chen, “The quantitative interpretation by measurement using the magnetic memory method (MMM)based on density functional theory," NDT \& E International, vol. 55, pp. 15-20, 2013.

[16] J. L. Ren, C. Chen, C. K. Liu et al., "Experimental research on microcosmic mechanism of stress-magnetic effect for magnetic memory testing," Journal of Aeronautical Materials, vol. 28, pp. 41-44, 2008.

[17] L. Dong, B. Xu, S. Dong et al., "Stress dependence of the spontaneous stray field signals of ferromagnetic steel," NDT and E International, vol. 42, no. 4, pp. 323-327, 2009.

[18] A. Lubk, S. Gemming, and N. A. Spaldin, "First-principles study of ferroelectric domain walls in multiferroic bismuth ferrite," Physical Review B - Condensed Matter and Materials Physics, vol. 80, no. 10, Article ID 104110, 2009.

[19] Y. KAkehashi and M. Patoary, "First-Principles Dynamical Coherent-Potential Approximation Approach to the Ferromagnetism of Fe, Co, and Ni," Journal of the Physical Society of Japan, vol. 11, pp. 1683-1694, 2011. 

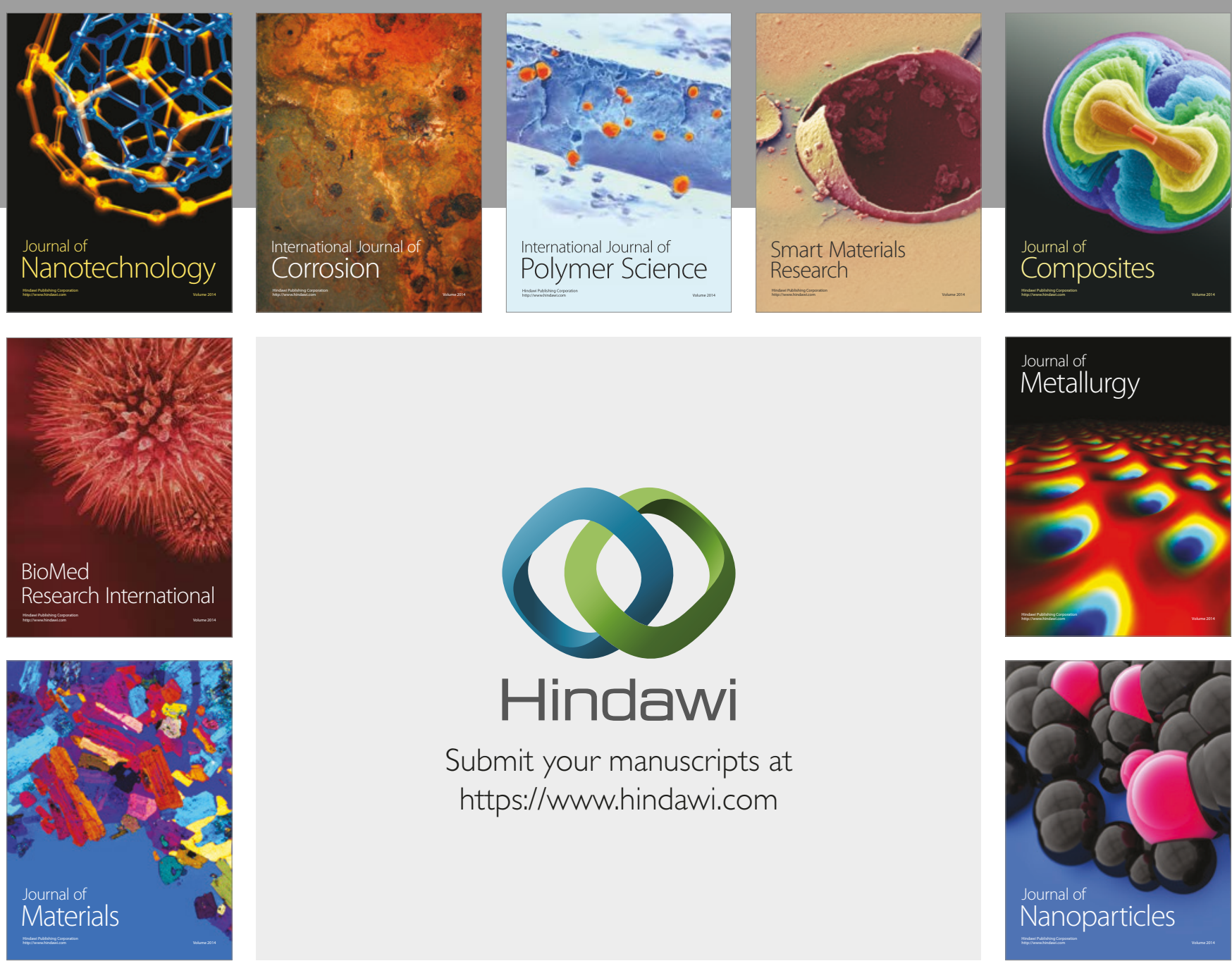

\section{Hindawi}

Submit your manuscripts at

https://www.hindawi.com
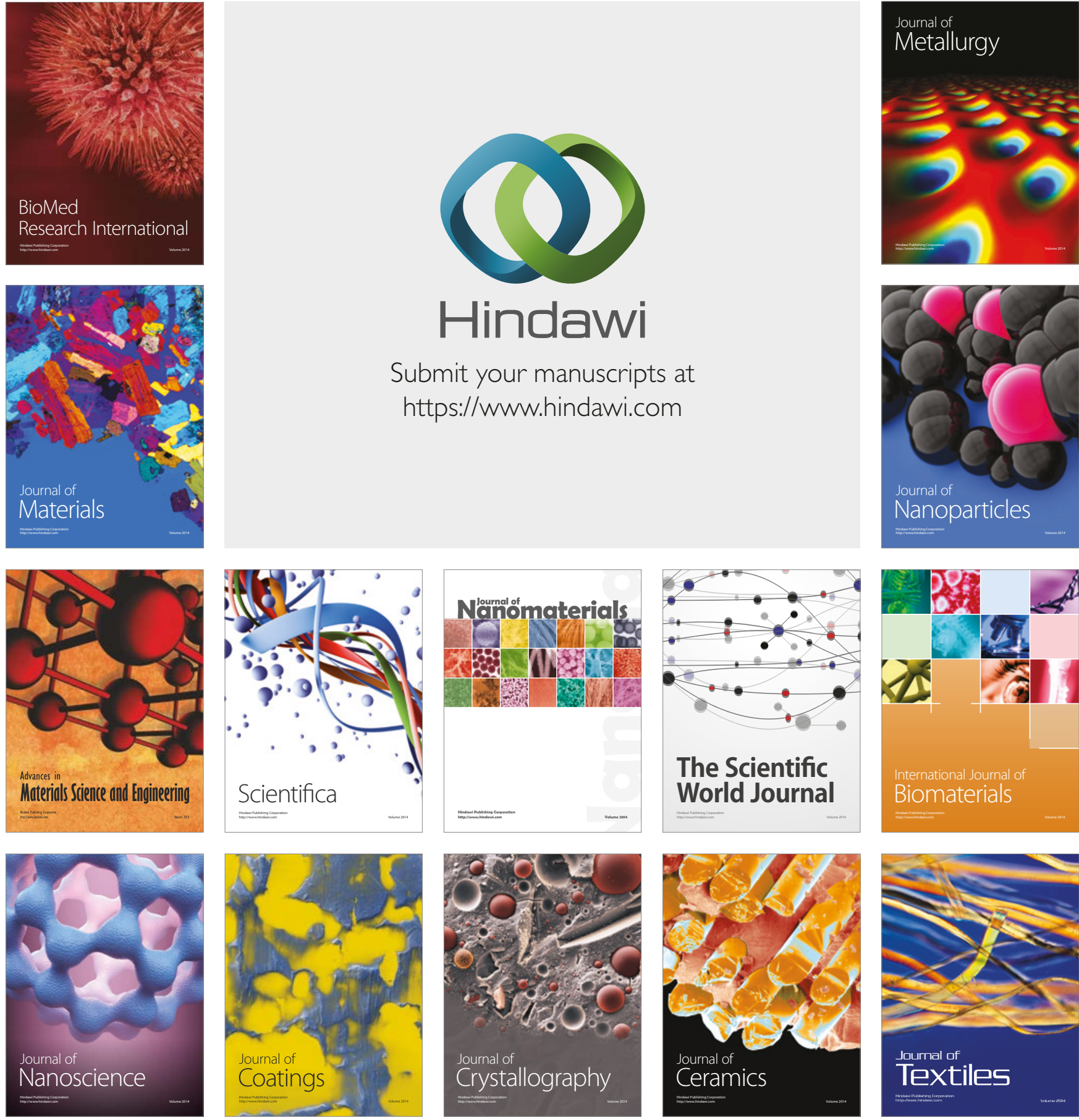

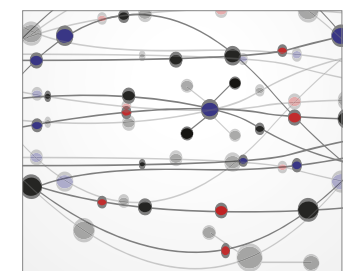

The Scientific World Journal
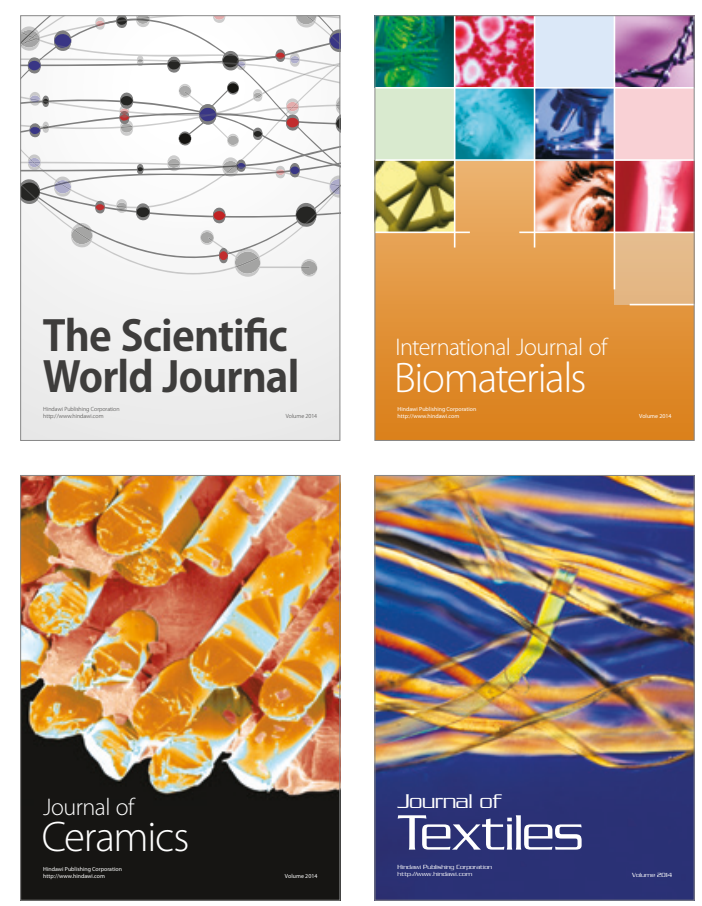